\title{
BRIEF COMMUNICATION Personalising adherence-enhancing interventions using a smart inhaler in patients with COPD: an exploratory cost- effectiveness analysis
}

Job F. M. van Boven (iD ${ }^{1,2}$, Breda Cushen (iD $^{3}$, Imran Sulaiman ${ }^{3}$, Garrett Greene ${ }^{3}$, Elaine MacHale ${ }^{3}$, Matshediso C. Mokoka ${ }^{3}$, Frank Doyle iD ${ }^{4}$, Richard B. Reilly ${ }^{5}$, Kathleen Bennett ${ }^{6}$ and Richard W. Costello ${ }^{3,7}$

Four inhaler adherence clusters have been identified using the INCA audio device in COPD patients: (1) regular use/good technique, (2) regular use/frequent technique errors, (3) irregular use/good technique, and (4) irregular use/frequent technique errors. Their relationship with healthcare utilization and mortality was established, but the cost-effectiveness of adherence-enhancing interventions is unknown. In this exploratory study, we aimed to estimate the potential cost-effectiveness of reaching optimal adherence in the three suboptimal adherence clusters, i.e., a theoretical shift of clusters 2,3 , and 4 to cluster 1 . Cost-effectiveness was estimated over a 5-year time horizon using the Irish healthcare payer perspective. We used a previously developed COPD health-economic model that was updated with INCA trial data and Irish national economic and epidemiological data. For each cluster, interventions would result in additional quality-adjusted life years gained at reasonable investment. Cost-effectiveness was most favorable in cluster 3, with possible cost savings of $€ 845 /$ annum/person.

npj Primary Care Respiratory Medicine (2018)28:24; doi:10.1038/s41533-018-0092-8

\section{INTRODUCTION}

In chronic obstructive pulmonary disease (COPD), real-world adherence to maintenance therapy can be as low as $20 \%{ }^{1}$ In contrast, trials report adherence rates of over $80 \%$ in most participants. ${ }^{2}$ Previous studies showed that patients with high adherence have significantly better clinical and economic outcomes. ${ }^{3}$ Consequently, interventions focusing on adherence enhancement have shown to be effective and cost-effective. ${ }^{4,5}$ Yet, in these studies, only average adherence was assessed and no distinction was made between the different aspects of nonadherence. Optimal implementation of inhaled therapy involves both regular use as well as good inhaler technique. ${ }^{6}$ In our previous work, we identified four distinct clusters of inhaler adherence that were associated with differential clinical outcomes. ${ }^{7}$ Economic evaluations of adherence-enhancing interventions in each of those clusters have not been performed, but could help prioritizing specific target populations for tailored interventions. The aim of this follow-up study is to estimate the potential cost-effectiveness of reaching optimal adherence in each suboptimal adherence cluster.

\section{RESULTS}

The study's baseline population characteristics $(n=226)$ have been described elsewhere. ${ }^{1,7}$ In short, cluster 1 had the lowest mortality during follow-up. Cluster 2 had the highest rate of antibiotic and/or oral steroids community prescriptions. The highest overall healthcare use was attributable to patients from cluster 3 . Cluster 4 had the highest mortality (Fig. 1).

\begin{abstract}
Cost-effectiveness
Cost-effectiveness analysis results are shown in Table 1. Adherence-enhancing interventions seem generally cost-effective, given all ICERs are below the Irish cost-effectiveness threshold of $€ 45,000$ /QALY. ${ }^{8}$ In other words, this means that all adherenceenhancing interventions would cost less than $€ 45,000$ to gain one life-year in perfect health. For each cluster, the theoretical intervention would result in additional life years and QALYs gained. Notably, for cluster 3 , an intervention would be costsaving (i.e., less costs and more QALYs).
\end{abstract}

\section{DISCUSSION}

We performed exploratory cost-effectiveness modeling to assess the potential economic benefits of improved adherence in three clusters of patients with suboptimal adherence. In all clusters, interventions seem cost-effective. Moreover, in patients with irregular use but good inhaler technique, a possible cost-saving of $€ 845$ /annum/person could be yielded despite the higher cost of medication arising from better adherence.

Remote monitoring of adherence on a longitudinal basis provides an accurate evaluation of regularity of use as well as inhaler technique. The use of a technology, such as the one

\footnotetext{
${ }^{1}$ Department of General Practice \& Elderly Care, University Medical Center Groningen, Groningen Research Institute for Asthma and COPD (GRIAC), University of Groningen, Groningen, The Netherlands; ${ }^{2}$ Department of Pharmacy, Unit of Pharmacoepidemiology \& Pharmacoeconomics, University of Groningen, Groningen, The Netherlands; ${ }^{3}$ Royal College of Surgeons in Ireland, Clinical Research Centre, Smurfit Building Beaumont Hospital, Dublin, Ireland; ${ }^{4}$ Department of Psychology, Division of Population Health Sciences,

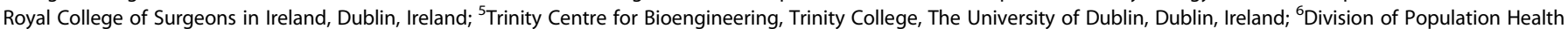
Sciences, Royal College of Surgeons in Ireland, Dublin, Ireland and ${ }^{7}$ Department of Respiratory Medicine, Royal College of Surgeons in Ireland, Dublin, Ireland Correspondence: Job FM van Boven (j.f.m.van.boven@rug.nl)
}

Received: 26 February 2018 Revised: 23 May 2018 Accepted: 23 May 2018

Published online: 27 June 2018 


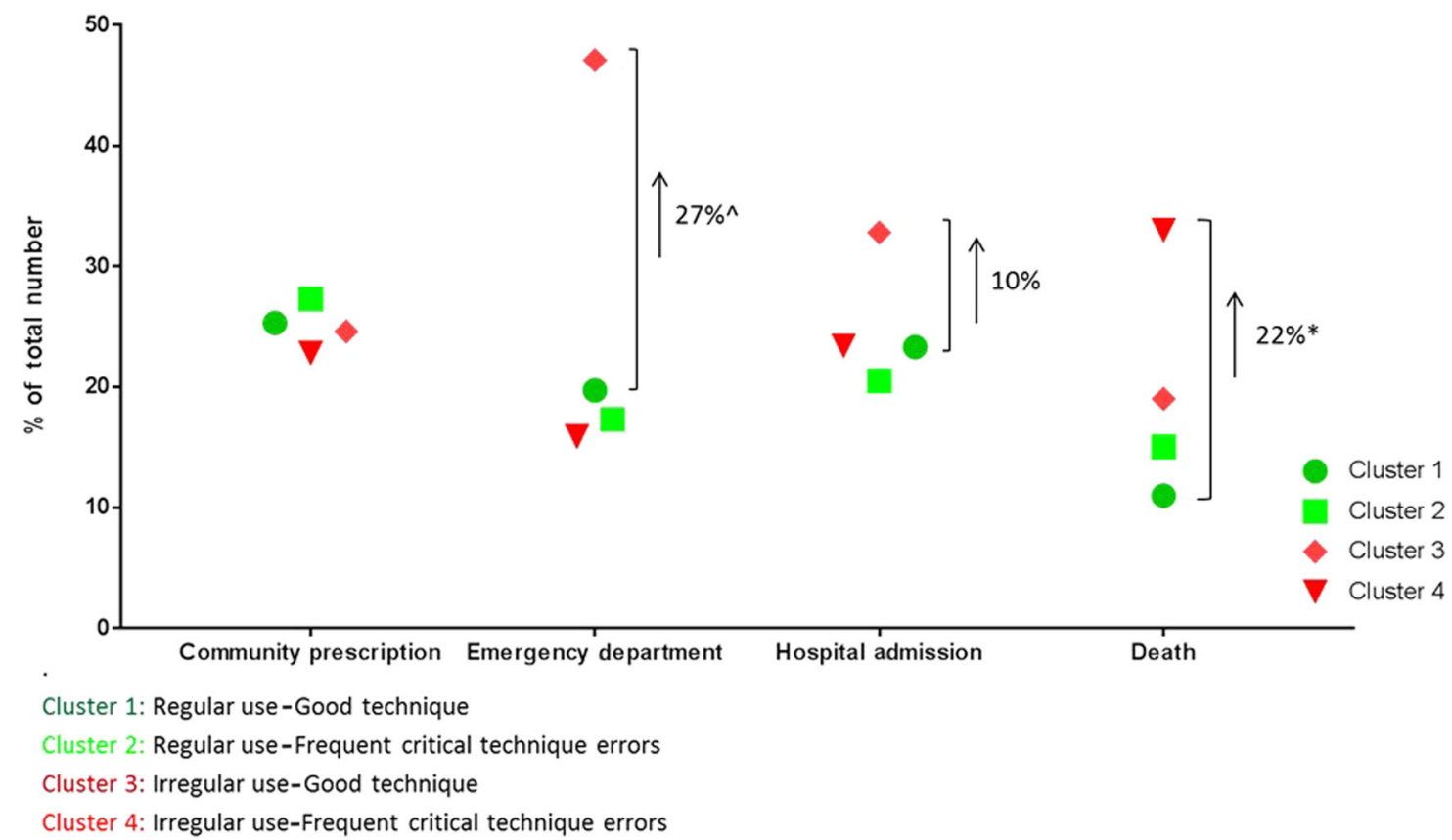

Fig. 1 Proportional contribution of each adherence cluster to all-cause clinical outcomes over the 12-month follow-up period (adjusted for the number of participants per cluster). Reported differences are the absolute differences in the proportion of events attributable to cluster 3 vs. cluster 1 for emergency department and hospital admission and cluster 4 vs. cluster 1 for death. $\wedge$ denotes $p=0.05,{ }^{*} p<0.05$

\begin{tabular}{|c|c|c|c|c|c|c|}
\hline \multicolumn{7}{|c|}{ Cluster 2: Regular use, frequent technique errors } \\
\hline Cluster 2 & $€ 10,150$ & $€ 0$ & $€ 2723$ & $€ 7428$ & 3.52 & 2.81 \\
\hline Difference & $€ 1235$ & $€ 184$ & $€ 179$ & $€ 872$ & 0.23 & 0.19 \\
\hline Intervention & $€ 11,812$ & $€ 184$ & $€ 2936$ & $€ 8692$ & 3.80 & 3.03 \\
\hline Cluster 3 & $€ 12,657$ & $€ 0$ & $€ 2395$ & $€ 10,262$ & 3.40 & 2.70 \\
\hline Difference & $-€ 845$ & $€ 184$ & $€ 541$ & $-€ 1570$ & 0.39 & 0.33 \\
\hline ICER & \multicolumn{6}{|c|}{ Cost-saving (i.e., less costs, more QALYs) } \\
\hline \multicolumn{7}{|c|}{ Cluster 4: Irregular use, frequent technique errors } \\
\hline ICER & \multicolumn{6}{|c|}{$€ 3935 /$ QALY gained } \\
\hline
\end{tabular}

ICER incremental cost-effectiveness ratio, QALY quality-adjusted life year

${ }^{a}$ Note that the mean per-patient intervention costs are slightly lower given some patients die within the first year and so do not cost the full $€ 200$

described in this report, identified four patterns of adherence and may give guidance as to how we might approach this challenge in a personalized manner. Hence, in the future, by providing personalized interventions to the highest risk groups we may both improve adherence and have a positive impact on both clinical and economic outcomes in those most in need, such as those in cluster 3 . The need for personalized adherenceenhancing interventions has recently been highlighted. ${ }^{9}$ They could include education and training on inhaler technique for those with frequent technique errors, patient reminders for those with irregular use due to forgetfulness, and shared decision- making and motivational interviewing for those with irregular use due to a conscious, intentional decision. ${ }^{9}$

This study is a first attempt to estimate the cost-effectiveness of interventions in different adherence clusters, but was limited by the use of post hoc and short-term effectiveness data for the different clusters. Regarding generalizability, absolute costs may differ per country or setting, but we expect relative cluster results to be comparable. Our exploratory cost-effectiveness model estimates should be confirmed when long-term clinical intervention trial data become available, including extensive sensitivity and scenario analyses as well as analyses in a non-admitted primary care COPD population. 


\section{CONCLUSION}

Personalized adherence interventions targeting patient-specific regularity of inhaler use and inhaler technique could result in clinical and economic benefits for COPD patients.

\section{METHODS}

Study design

This was an exploratory cost-effectiveness analysis. Ethical approval for the clinical study was obtained from the Beaumont Hospital Ethics Committee.

\section{Patient population}

Patient selection and data collection has been described previously. Briefly, hospitalized patients with COPD, prescribed salmeterol/fluticasone propionate (Seretide ${ }^{\circledR}$, GlaxoSmithKline, Ireland), were included and written informed consent was obtained from all participants.

Inhaler adherence

Adherence was assessed using the INCA ${ }^{\mathrm{TM}}$ device that can track both timing and quality of inhaler use. ${ }^{1}$ The INCA acoustic recordings allowed for identification of the following four adherence clusters: ${ }^{7}(1)$ regular use/ good technique, (2) regular use/frequent technique errors, (3) irregular use/good technique, and (4) irregular use/frequent technique errors. Cluster-specific healthcare utilization and mortality was studied previously ${ }^{7}$ and is summarized in Fig. 1.

\section{Cost-effectiveness analysis}

The cost-effectiveness of enhanced adherence, i.e., the theoretical shift of patients from poor adherence (i.e., cluster 2,3 , or 4 ) to good adherence (i.e., cluster 1), was estimated. Therefore, a previously developed, described, and validated health-economic mode $l^{5}$ was used and populated with Irish and INCA-specific cost (for medication, intervention, and healthcare utilization) and effect (mortality and exacerbations) data (Appendix 1). The model has received a high-quality score in the latest COPD model review, ${ }^{10}$ and estimates the incremental cost-effectiveness ratio (ICER) in terms of costs $(2013, €)$ per quality-adjusted life-year (QALY) gained from the Irish healthcare payer's perspective assuming fixed perpatient intervention costs of $€ 200$ (authors' estimation). Three ICERs were calculated using cluster 1 as intervention and clusters 2, 3, and 4, respectively, as usual care scenarios. In line with recommendations, ${ }^{11}$ a policy-relevant time horizon of 5 years was used, taking into account a $5 \%$ discount rate for both costs and effects as per Irish Health Information and Quality Authority guidelines (www.hiqa.ie).

Data availability statement

Detailed data and characteristics of the study population are available in ref. ${ }^{1}$ and other data that support the findings of this study are available from the corresponding author on reasonable request.

\section{AUTHOR CONTRIBUTIONS}

J.F.M.v.B. and R.W.C. designed the study. I.S., M.M., and E.M. were primarily involved in patient recruitment and management of the patients, R.B.R. was primarily involved in the audio analysis required for this manuscript. B.C., G.G., and K.B. were primarily involved in data collection and analysis. J.F.M.v.B. was primarily involved in the health-economic analysis and wrote the first draft of the manuscript. All authors provided input and approved the final manuscript. J.F.M.v.B. is the guarantor of the study.FundingThis was a researcher-initiated study, funded by the Health Research
Board of Ireland, Clinical Scientist Award (RC), and the Irish Research Council (REPRO/ 2015/90). The funders of the study had no role in the study design, data collection, data analysis, data interpretation, or writing of the report.

\section{ADDITIONAL INFORMATION}

Supplementary Information accompanies the paper on the npj Primary Care Respiratory Medicine website (https://doi.org/10.1038/s41533-018-0092-8).

Competing interests: R.W.C. and R.B.R. are named on a patent for the INCA device.

Publisher's note: Springer Nature remains neutral with regard to jurisdictional claims in published maps and institutional affiliations.

\section{REFERENCES}

1. Sulaiman, I. et al. Objective assessment of adherence to inhalers by patients with chronic obstructive pulmonary disease. Am. J. Respir. Crit. Care Med. 195, 1333-1343 (2017).

2. Vestbo, J. et al. Adherence to inhaled therapy, mortality and hospital admission in COPD. Thorax 64, 939-943 (2009).

3. van Boven, J. F. et al. Clinical and economic impact of non-adherence in COPD: a systematic review. Respir. Med. 108, 103-113 (2014).

4. Klijn, S. et al. Effectiveness and success factors of educational inhaler technique interventions in asthma \& COPD patients: a systematic review. NPJ Prim. Care Respir. Med. 27, 24 (2017).

5. van Boven, J. F. et al. Improving inhaler adherence in patients with chronic obstructive pulmonary disease: a cost-effectiveness analysis. Respir. Res. 15, 66 (2014).

6. Vrijens, B. et al. What we mean when we talk about adherence in respiratory medicine. J. Allergy Clin. Immunol. Pract. 4, 802-812 (2016).

7. Cushen, $B$, et al. The clinical impact of different adherence behaviours in patients with severe COPD. Am. J. Respir. Crit. Care Med. https://doi.org/10.1164/ rccm.201712-2469LE (2018).

8. O'Mahony, J. F. \& Coughlan, D. The Irish cost-effectiveness threshold: does it support rational rationing or might it lead to unintended harm to Ireland's health system? Pharmacoeconomics 34, 5-11 (2016).

9. Van Boven, J. F. et al. Urging Europe to put non-adherence to inhaled respiratory medication higher on the policy agenda: a report from the First European Congress on Adherence to Therapy. Eur. Respir. J. 49, 170076 (2017).

10. Zafari, Z. et al. A systematic review of health economics simulation models of chronic obstructive pulmonary disease. Value Health 20, 152-162 (2017).

11. van der Schans, S. et al. Systematic review and quality appraisal of costeffectiveness analyses of pharmacologic maintenance treatment for chronic obstructive pulmonary disease: methodological considerations and recommendations. Pharmacoeconomics 35, 43-63 (2017).

Open Access This article is licensed under a Creative Commons Attribution 4.0 International License, which permits use, sharing, adaptation, distribution and reproduction in any medium or format, as long as you give appropriate credit to the original author(s) and the source, provide a link to the Creative Commons license, and indicate if changes were made. The images or other third party material in this article are included in the article's Creative Commons license, unless indicated otherwise in a credit line to the material. If material is not included in the article's Creative Commons license and your intended use is not permitted by statutory regulation or exceeds the permitted use, you will need to obtain permission directly from the copyright holder. To view a copy of this license, visit http://creativecommons. org/licenses/by/4.0/.

(C) The Author(s) 2018 